\title{
A non-local plasticity theory for slow granular flows
}

\author{
Prabhu R. Nott ${ }^{*}$ \\ Department of Chemical Engineering, Indian Institute of Science, Bangalore 560012, India
}

\begin{abstract}
Motivated by experimental observations of apparent non-locality in the mechanical response of granular materials, a few recent studies have proposed non-local rheological models. Though their predictions agree with some aspects of experimental observations, the tenets of the models are not experimentally verified, nor do they have an experimentally verifiable or plausible mechanical basis. Here we present a non-local model that is based on the simple idea of volume averaging, and show that it overcomes many of the shortcomings of the previously proposed models, and is superior in its predictive capabilities.
\end{abstract}

\section{Introduction}

A few recent studies on the mechanics of dense granular materials have shown evidence of non-locality in the mechanical response, wherein the motion of an intruder is seemingly aided by shearing the granular material far from it $[1,2]$. This behaviour is not explained by classical rate-independent plasticity theories; indeed, these theories have the more serious failings of being unable to predict the kinematics of simple shear flows, and being unable to account for the universally observed feature of deformation-induced dilation at constant pressure. Nonlocal theories have been proposed in the past, but they are of largely phenomenological origin [3,4], or based on the introduction of an additional field variable obeying a conservation equation whose mechanical origin is debatable [5]. In this work, we present a non-local, rateindependent plasticity theory whose mechanical origin is easy to comprehend, and involves no additional field variables - it captures rather simply the physical picture of plastic events in a spatial point influencing its neighbourhood. Moreover, it is based on the widely accepted method of coarse-graining that is used in multiphase flows, and for complex fluids with internal structure. The theory predicts the kinematics of simple shear flows, such as the exponentially decaying (or saturating) velocity profile in simple shear, and shearinduced dilatancy. Finally, we show that our non-local theory plasticity theory is Hadamard well posed, a significant improvement over the previous theories.

\section{Local plasticity theory}

Before describing non-local plasticity models, it is useful to very briefly outline local critical state plasticity theory. The theory provides a relation for the stress when the material is in a state of plastic deformation. The two elements of the theory are the yield condition,

$$
F(\boldsymbol{\sigma}, v) \equiv \tau\left(\boldsymbol{\sigma}^{\prime}\right)-p_{c}(v) \sin \phi Y(\alpha, v)=0,
$$

where $\alpha \equiv p / p_{c}(v)$, and the flow rule,

$$
D_{\mathrm{ij}}=\lambda \partial G / \partial \sigma_{\mathrm{ji}}
$$

In the above, the yield function $F$ and the plastic potential $G$ are scalar functions of the Cauchy stress $\sigma, Y$ is a function of the pressure $p$ and the solids fraction $v, \tau$ is a scalar function of the deviator stress $\sigma^{\prime} \equiv \boldsymbol{\sigma}-p \boldsymbol{I}, \boldsymbol{D}$ is the rate of deformation tensor, and $\lambda$ is fluidity variable. The quantity $p_{c}(v)$ represents the pressure at the critical state, a state of isochoric deformation.

A simple example of classical plasticity is the extended von Mises yield condition [6,7]

$$
\tau=\left(\sigma_{\mathrm{ij}} \sigma_{\mathrm{ji}}\right)^{1 / 2}, \quad Y=\left(\mathrm{n} \alpha-(\mathrm{n}-1) \alpha^{\mathrm{n} /(\mathrm{n}-1)}\right),
$$

and the associated flow rule $G=F$, for which (1) and (2) may be solved to yield the following form for the stress [7],

$$
\boldsymbol{\sigma} \equiv p_{c}(v)\left(1+\mu_{b} \boldsymbol{\nabla} \cdot \mathbf{u} / \lambda\right)^{\mathrm{n}-1}+2 \mu Y(\alpha, v) D_{\mathrm{ij}} / \lambda,
$$

where $\lambda=-\gamma \equiv\left(D_{\mathrm{ij}}^{\prime} D_{\mathrm{ji}}^{\prime}\right)^{1 / 2}$ is a scalar measure of the rate of deformation, $n$ is a constant such that $\mathrm{n}>1, \mathrm{n}-1 \ll 1$, and $\mu_{b} \equiv 1 /(n \sin \phi), \mu \equiv \sin \phi$ are the bulk and shear plastic moduli.

As already stated, classical plasticity has three important deficiencies [4,8]: (i) it does not predict the velocity field in simple shear flows, (ii) it does not capture dilation in in simple shear flows, and (iii) it is Hadamard ill-posed.

\footnotetext{
Corresponding author: prnott@,chemeng.iisc.ernet.in
} 


\section{Previous non-local plasticity models}

Mohan et al. [4] proposed the Cosserat plasticity model to address some of the deficiencies of classical plasticity. The tenets of a Cosserat continuum are that the Cauchy stress is not necessarily symmetric, and the balance of angular momentum must be enforced. The theory introduces the additional kinematic variable of the mean particle spin $\boldsymbol{\omega}$ (which in a classical continuum is equal to the vorticity $\mathbf{w}$ ), and the additional dynamic variable $\mathbf{M}$, called the couple stress tensor, which is simply the angular momentum flux. With the following simple modifications of the extended von Mises yield condition (3) and the flow rule (2)

$$
\begin{gathered}
\tau=\left(\mathrm{a}_{1} \sigma_{\mathrm{ij}} \sigma_{\mathrm{ji}}+\mathrm{a}_{2} \sigma_{\mathrm{ij}} \sigma_{\mathrm{i}}+l^{2} M_{\mathrm{ij}} M_{\mathrm{ji}}\right)^{1 / 2} \\
D_{\mathrm{ij}}+\epsilon_{\mathrm{ijk}}\left(\omega_{\mathrm{k}}-\mathrm{w}_{\mathrm{k}}\right)=\lambda \partial F / \partial \sigma_{\mathrm{ji}}, \partial \omega_{\mathrm{i}} / \partial x_{\mathrm{j}}=\lambda \partial F / \partial M_{\mathrm{ji}}
\end{gathered}
$$

they showed that the model captures several observed features of the kinematics within shear layers, including the exponential decay (or saturation) of the velocity within the shear layer in simple shear, the weak dependence of the shear layer thickness on the system size, and the deviation between $\boldsymbol{\omega}$ and $\mathbf{w}$. These features are a consequence of the mesoscopic length scale $l$ that is present in the model. The drawback of the model is that experiments have thus far not been able to measure the couple stress, or confirm the asymmetry of the Cauchy stress, which are important elements of the model.

More recently, another non-local theory has been proposed [5], wherein the central tenet is that the constitutive relation (4) must be supplemented by a Helmholtz equation for the fluidity,

$$
l^{2} \nabla^{2} \lambda=\left(\lambda-\lambda_{\text {loc }}\right)
$$

where $\lambda_{\text {loc }} \equiv-\gamma$ is the "local" fluidity. This model too incorporates a mesoscopic length scale $l$, and has been shown to capture the kinematics in simple shear, and its predictions are in consonance with experimental observations of more complex flows. The drawbacks of this model are: (i) the basis for (7) is tenuous, and uses a boundary condition for $\lambda$ that is unconvincing, (ii) the model does not capture the important phenomenon of dilatancy, and (iii) it does not escape the Hadamard illposedness of classical pasticity.

\section{A new non-local model}

We propose a model that repairs the aforementioned drawbacks of the previously proposed non-local models. The basis of the model lies in volume averaging the flow rule, an idea that follows from the intuitive expectation that plastic deformation at a point must be influenced by the stress state not simply at that point alone, but also in its neighbourhood (Fig. 1). This intuitive idea is in agreement with the now widely recognized fact that stress in granular materials is transmitted along quasilinear clusters of grains called force chains - hence plastic deformation at one point can induce deformation at a distance from it due to reformation of the chains. With this in mind, we propose that the flow rule (2) (with $G=F$ ), be replaced by

$$
D_{\mathrm{ij}}=\int_{y} g(\boldsymbol{x}-\boldsymbol{y})\left[\lambda \partial F / \partial \sigma_{\mathrm{ji}}\right] \mathrm{d} \boldsymbol{y},
$$

where $g(\boldsymbol{x}-\boldsymbol{y})$ is a weight function satisfying the properties

$$
\int_{y} g(x-y) \mathrm{d} \mathbf{y}=1, \quad \int_{y} g(x-y)|x-y|^{2} \mathrm{~d} \mathbf{y}=l^{2},
$$

where $l$ is the radius of the averaging volume. Using the extended von Mises yield condition (3) for $F$ in (8), expanding the term within square brackets in a Taylor series about $\boldsymbol{y}=\boldsymbol{x}$, and solving for $\boldsymbol{\sigma}$ as a perturbation series expansion in $l^{2}$, we get

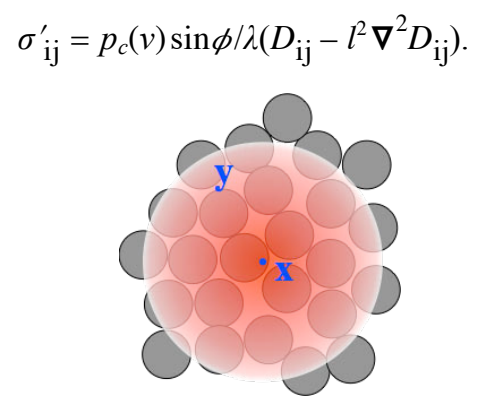

Fig. 1: Schematic diagram of the volume averaging of the flow rule about the point $\boldsymbol{x}$.

Substituting (10) in the yield condition, we get the relation for the fluidity

$$
\lambda=-\gamma-l^{2} \nabla^{2} \gamma
$$

Note the similarity between (11) and (7), and also the important difference that (11) is explicit in $\lambda$ - it does not require a boundary condition. Moreover, the volume averaging process provides a clearer and physically acceptable process by which the mesoscopic length scale $l$ is introduced into the constitutive equation for the stress. Most importantly, non-locality is reflected not just in $\lambda$, but also in the higher gradients in $\boldsymbol{D}$ in the relation for $\sigma$. We note that the local flow rule is based on maximization of the plastic work - our proposed nonlocal flow rule is a simple extension, in the sense that the plastic work is maximized over a volume of dimension $l$, but not necessarily at every point.

It is straightforward to show that the model constitutive (10)-(11) captures all the kinematic features of slow granular flows that are captured by the previous non-local models, described in section 2. However, it has the following advantages over the previous models: (i) it does not require the solution of a separate differential equation for the fluidity $\lambda$, therefore circumventing the requirement for boundary conditions, (ii) it brings in non-locality in an intuitively acceptable manner, that is in consonance with experimental observations [1], and (iii) the model is unconditionally well posed in the sense of Hadamard. 
Funding from the Science and Engineering Research Board, India is gratefully acknowledged.

\section{References}

1. K. A. Reddy, Y. Forterre, O. Pouliquen, Phys. Rev. Lett. 106, 108301 (2011).

2. K. Nichol, A. Zanin, R. Bastien, E. Wandersman, M. van Hecke, Phys. Rev. Lett. 104, 078302 (2010).

3. I. Vardoulakis, E. C. Aifantis, Acta Mech. 87, 197217 (1991).

4. L. S. Mohan, K. K. Rao, and P. R. Nott, J. Fluid Mech. 457, 377-409 (2002).

5. K. Kamrin, G. Koval, Phys. Rev. Lett. 108, 178301 (2012).

6. K. K. Rao, P. R. Nott, An Introduction to Granular Flown (Cambridge University Press, New York, 2008).

7. P. R. Nott, Acta Mech. 205, 151-160 (2009).

8. T. Barker, D. G. Schaeffer, P. Bohorquez, J. M. N. T. Gray, J. Fluid Mech. 779, 794-818 (2015). 